\section{(C) OPEN ACCESS}

Handling editor Tore K Kvien

- Additional material is published online only. To view please visit the journal online (http://dx.doi.org/10.1136/ annrheumdis-2016-210324)

For numbered affiliations see end of article.

\section{Correspondence to} Dr Michael J Ombrello, Translational Genetics and Genomics Unit, Office of the Clinical Director, Intramural Research Program, National Institute of Arthritis and Musculoskeletal and Skin Diseases, National Institutes of Health, US Department of Health and Human Services, 10 Center Drive, 12N248A, Building 10, MSC1560, Bethesda, MD 20852, USA; Michael.Ombrello@nih.gov

Received 5 August 2016 Revised 27 October 2016 Accepted 12 November 2016 Published Online First 7 December 2016

\section{CrossMark}

To cite: Ombrello MJ, Arthur VL, Remmers EF, et al. Ann Rheum Dis 2017:76:906-913.

\title{
Genetic architecture distinguishes systemic juvenile idiopathic arthritis from other forms of juvenile idiopathic arthritis: clinical and therapeutic implications
}

\author{
Michael J Ombrello, ${ }^{1}$ Victoria L Arthur, ${ }^{1}$ Elaine F Remmers, ${ }^{2}$ Anne Hinks, ${ }^{3}$ \\ loanna Tachmazidou, ${ }^{4}$ Alexei A Grom, ${ }^{5,6}$ Dirk Foell, ${ }^{7}$ Alberto Martini, ${ }^{8,9}$ \\ Marco Gattorno, ${ }^{9}$ Seza Özen, ${ }^{10}$ Sampath Prahalad, ${ }^{11,12}$ Andrew S Zeft, ${ }^{13}$
} John F Bohnsack, ${ }^{14}$ Norman T Ilowite, ${ }^{15}$ Elizabeth D Mellins, ${ }^{16}$ Ricardo Russo, ${ }^{17}$ Claudio Len, $^{18}$ Maria Odete E Hilario, ${ }^{18}$ Sheila Oliveira, ${ }^{19}$ Rae S M Yeung, ${ }^{20,21,22}$ Alan M Rosenberg, ${ }^{23}$ Lucy R Wedderburn, ${ }^{24,25}$ Jordi Anton, ${ }^{26}$ Johannes-Peter Haas, ${ }^{27}$ Angela Rosen-Wolff, ${ }^{28}$ Kirsten Minden, ${ }^{29,30}$ Klaus Tenbrock, $^{31}$ Erkan Demirkaya, ${ }^{10}$ Joanna Cobb, ${ }^{3,32}$ Elizabeth Baskin, ${ }^{1}$ Sara Signa, ${ }^{8}$ Emily Shuldiner, ${ }^{1}$ Richard H Duerr, ${ }^{33,34}$ Jean-Paul Achkar, ${ }^{35,36}$ M Ilyas Kamboh, ${ }^{34}$ Kenneth M Kaufman, ${ }^{5,6}$ Leah C Kottyan, 5,6 Dalila Pinto, ${ }^{37}$ Stephen W Scherer, ${ }^{38}$ Marta E Alarcón-Riquelme, ${ }^{39,40}$ Elisa Docampo, ${ }^{41,42}$ Xavier Estivill, ${ }^{42,43}$ Ahmet Gül, ${ }^{44}$ British Society of Pediatric and Adolescent Rheumatology (BSPAR) Study Group, Inception Cohort of Newly Diagnosed Patients with Juvenile Idiopathic Arthritis (ICON-JIA) Study Group, Childhood Arthritis Prospective Study (CAPS) Group, Randomized Placebo Phase Study of Rilonacept in sJIA (RAPPORT) Investigators, Sparks-Childhood Arthritis Response to Medication Study (CHARMS) Group, Biologically Based Outcome Predictors in JIA (BBOP) Group Carl D Langefeld, ${ }^{45}$ Susan Thompson, ${ }^{5,6}$ Eleftheria Zeggini, ${ }^{4}$ Daniel L Kastner, ${ }^{2}$ Patricia Woo, ${ }^{25}$ Wendy Thomson ${ }^{3,32}$

\section{ABSTRACT}

Objectives Juvenile idiopathic arthritis $(J A)$ is a heterogeneous group of conditions unified by the presence of chronic childhood arthritis without an identifiable cause. Systemic JIA (sJIA) is a rare form of JIA characterised by systemic inflammation. sJIA is distinguished from other forms of JIA by unique clinical features and treatment responses that are similar to autoinflammatory diseases. However, approximately half of children with sJIA develop destructive, long-standing arthritis that appears similar to other forms of JIA. Using genomic approaches, we sought to gain novel insights into the pathophysiology of sJIA and its relationship with other forms of JIA.

Methods We performed a genome-wide association study of 770 children with sJIA collected in nine countries by the International Childhood Arthritis Genetics Consortium. Single nucleotide polymorphisms were tested for association with sJIA. Weighted genetic risk scores were used to compare the genetic architecture of sJIA with other JIA subtypes.

Results The major histocompatibility complex locus and a locus on chromosome 1 each showed association with sJIA exceeding the threshold for genome-wide significance, while 23 other novel loci were suggestive of association with sJIA. Using a combination of genetic and statistical approaches, we found no evidence of shared genetic architecture between sJIA and other common JIA subtypes.

Conclusions The lack of shared genetic risk factors between SJIA and other JIA subtypes supports the hypothesis that $s J A$ is a unique disease process and argues for a different classification framework. Research to improve sJIA therapy should target its unique genetics and specific pathophysiological pathways.

\section{INTRODUCTION}

Juvenile idiopathic arthritis (JIA) encompasses a heterogeneous group of chronic childhood arthritides that develop without identifiable cause and last more than 6 weeks. ${ }^{1}{ }^{2}$ Children with JIA are placed into seven mutually exclusive categories based on clinical presentation: oligoarticular arthritis (oligoJIA) affects four or fewer joints; rheumatoid factor (RF)-negative polyarthritis (RF-polyJIA) involves five or more joints; RF-positive polyarthritis (RF+ polyJIA) is analogous to adult rheumatoid arthritis; psoriatic arthritis (PsA) is an arthritis that accompanies psoriasis; enthesitis-related arthritis encompasses non-PsA childhood spondyloarthropathy; systemic arthritis (sJIA, previously known as Still's disease) is characterised by prominent systemic inflammation and has a rare adult-onset 
counterpart; ${ }^{3}$ and undifferentiated arthritis includes arthritis that does not fit into any single category. ${ }^{12}$

sJIA is among the most severe childhood inflammatory diseases. First described by Sir George Frederic Still over a century ago, sJIA is marked by arthritis and systemic inflammation with quotidian fever, evanescent salmon pink skin rash, lymphadenopathy, hepatosplenomegaly and serositis. ${ }^{2}{ }^{4}$ It is frequently complicated by macrophage activation syndrome, a potentially lethal form of hemophagocytic lymphohistiocytosis. ${ }^{5}$ Although sJIA only constitutes approximately $10 \%$ of JIA in populations of European descent, ${ }^{15}$ its disproportionately large share of the morbidity and mortality observed in $\mathrm{JIA}^{6}$ underscores the importance of understanding and targeting its root causes.

The unique clinical characteristics of sJIA suggest that it is distinct from other forms of JIA, leading to the contention by some that sJIA should be separated from other forms of JIA and labelled as an autoinflammatory disease. ${ }^{7}$ This has been challenged by identification of autoantibodies in some patients with sJIA. ${ }^{8}$ Furthermore, while the systemic inflammatory features of sJIA seem to distinguish it from other forms of JIA, most children with sJIA eventually shed these features, leaving up to half of children with a persistent form of arthritis that is similar to the oligoarticular and polyarticular forms of JIA. ${ }^{59}$ Finally, significant differential effects of anticytokine agents have been observed between sJIA and other forms of JIA. ${ }^{10}$ However, due to the highly variable therapeutic responses to each agent in sJIA, this has not concretely advanced our understanding of how sJIA mechanistically relates to other forms of JIA.

One approach to evaluate the similarity of diseases is to examine shared pathophysiology through statistical comparisons of disease-specific genetic association data. ${ }^{11}$ For example, studies of inflammatory bowel disease and spondyloarthritis have identified shared genetic risk factors, providing rationale for similar treatment choices. ${ }^{11}$ In JIA, the majority of genetic and genomic investigations have focused on the combination of the most common subtypes, oligoJIA and RF-polyJIA (henceforth referred to in this manuscript as polygoJIA), ${ }^{12}{ }^{13}$ but until recently, ${ }^{14}$ because of insufficient numbers of patients with sJIA, there have been only underpowered genetic studies and no genome-wide studies of sJIA. Comparisons of the genomic underpinnings of sJIA relative to other forms of JIA have therefore also been lacking.

To gain insight into the pathogenesis of sJIA, we established the International Childhood Arthritis Genetics (INCHARGE) consortium. Together, we gathered the largest sJIA study population ever assembled, which included 982 children from nine countries on three continents. Using this collection, we performed the first genome-wide association study (GWAS) of sJIA. We recently reported the results of our intensive examination of the major histocompatibility complex (MHC) locus in this study population, which identified the class II human leucocyte antigen (HLA) region as a strong sJIA susceptibility locus. ${ }^{14}$ Here, we report the findings of the GWAS, beyond the MHC locus. Using the GWAS results, we have performed the first direct comparison of the genetic architecture of sJIA with those of the most common forms of JIA.

\section{METHODS}

\section{Study design and participants}

Peripheral blood specimens were collected from children diagnosed with sJIA according to the International League of Associations for Rheumatology (ILAR) criteria ${ }^{2}$ by paediatric rheumatologists at participating medical centres in nine countries (see online supplementary text and figure S1). Blood samples were also obtained from geographically matched control subjects. In addition, single nucleotide polymorphism (SNP) genotype data from geographically matched control populations were used, when available. The INCHARGE project was granted institutional review board (IRB) approval by the University of Manchester. Subjects were enrolled in accordance with all local ethics regulations, with the approval of local IRBs at each contributing medical centre, and with informed parental consent.

\section{Genotyping, quality control and imputation}

Genomic DNA was extracted from peripheral blood samples. Samples were genotyped at the National Human Genome Research Institute (Bethesda, Maryland, USA) using Human Omni1M arrays (Illumina) in accordance with the manufacturer's protocols. SNP genotype data were stratified by country of origin and rigorous quality control (QC) operations were undertaken separately in each case and control population, as previously reported. ${ }^{14}$ Principal components analysis and multidimensional scaling were used in each geographically defined case-control collection to generate nine ancestrally matched case-control strata, as previously described. ${ }^{14}$ Genomic control inflation factors were calculated, per stratum, as an objective metric of ancestral matching. ${ }^{14}$ An overview of the QC parameters is shown in online supplementary figure S2, and complete details are provided in the online supplementary text and our previous publication. ${ }^{14}$

SNP genotypes were phased using IMPUTE2 $2{ }^{15}$ and SNP imputation was performed separately for each geographically defined stratum using IMPUTE2 software and the multiancestral 1000 Genomes Project dataset (phase III) as the reference population. ${ }^{16}$ Genotype probabilities for common markers (case minor allele frequency $\geq 0.04$ ) that were imputed with high quality (info scores $\geq 0.8$ ) were included in subsequent analyses.

\section{Statistical analysis}

Association testing of genotype probabilities was performed using logistic regression in each geographically defined stratum with SNPTESTv $2,{ }^{15}$ adjusting for gender and ancestry informative principal components. Association results were meta-analysed using GWAMA. ${ }^{17}$ Heterogeneity was evaluated in the meta-analyses using the $\mathrm{I}^{2}$ statistic. Weighted genetic risk scores (wGRSs) were calculated and receiver operator characteristic (ROC) curve analyses were performed according to the method of Karlson et al. ${ }^{18}$ wGRSs were calculated as the sum of the risk allele counts, weighted by the natural logarithm of the OR. The wGRS for polygoJIA (polygo-wGRS) incorporated 23 independent risk alleles reported by Hinks et al ${ }^{12}$ (see online supplementary table S1). The wGRS for RF+polyJIA (RF +poly-wGRS) was based on the RF+ polyJIA-associated wGRS- $11^{19}$ (see online supplementary table S2). The case and control distributions of risk alleles and wGRSs were evaluated with the Wilcoxon rank-sum test. Association of wGRSs with sJIA was tested by logistic regression, adjusted for ancestry and gender. The ability of wGRSs to discriminate between sJIA and other JIA subtypes was evaluated with ROC curve analysis and calculation of the area under the curve (AUC) using R. Quantile-quantile $(\mathrm{Q}-\mathrm{Q})$ plots were generated using the sJIA association data, conditional on sets of polygoJIA-associated SNPs, ${ }^{12}$ as previously described. ${ }^{20}$

\section{RESULTS}

We performed SNP genotyping of 1413 children, including 982 children with sJIA and 431 healthy children. SNP genotype data, in silico, were incorporated from five existing control 
populations, including 7579 additional subjects, producing a total study population of 8992 individuals. After stringent QC, 770 patients with sJIA and 6947 control subjects were stratified into nine geographically defined and ancestrally matched casecontrol collections (table 1, see online supplementary text and tables S3 and S4), as previously described. ${ }^{14}$ Because most in silico control datasets were generated using SNP genotyping platforms different from that used in our study, the final number of SNPs evaluated in strata with in silico data was reduced to the intersection of the different SNP arrays (see online supplementary text and table S4). Imputation produced sets of between 4147566 and 6832892 imputed SNPs that

Table 1 Summary of SNP datasets from nine sJIA case-control collections after quality control operations

\begin{tabular}{lcclll}
\hline Stratum & Cases & Controls & $\begin{array}{l}\text { Genotyped } \\
\text { SNPs (filtered) }\end{array}$ & Imputed & $\begin{array}{l}\text { Imputed } \\
\text { SNPs } \\
\text { (filtered) }\end{array}$ \\
\hline USA & 243 & 1718 & 476196 & 18263974 & 6189397 \\
\hline UK & 202 & 4097 & 440688 & 18263701 & 6255387 \\
\hline Germany & 115 & 193 & 682516 & 18266121 & 6391432 \\
\hline Turkey & 49 & 94 & 682598 & 18270612 & 6389103 \\
Italy & 49 & 59 & 686397 & 18269173 & 6375260 \\
\hline Brazil & 48 & 62 & 740509 & 18263563 & 6698947 \\
Argentina & 33 & 115 & 659100 & 18263401 & 6129601 \\
\hline Canada & 17 & 427 & 396935 & 18263146 & 5812530 \\
Spain & 14 & 182 & 156136 & 18261199 & 4147550 \\
\hline Total & 770 & 6947 & & &
\end{tabular}

sJIA, systemic juvenile idiopathic arthritis; SNP, single nucleotide polymorphism. passed postimputation QC processes (see online supplementary text). Association results were combined by fixed-effect meta-analysis, producing meta-analytic association data for 5600610 SNPs (figure 1). This analysis identified two sJIA susceptibility loci with associations exceeding the threshold for genome-wide significance, adjusted for the two models tested $\left(\mathrm{p}<2.5 \times 10^{-8}\right)$, and 23 loci with highly suggestive evidence of association $\left(\mathrm{p}<5 \times 10^{-6}\right.$; table 2$)$. With the exception of the MHC locus none of these loci have been previously implicated in sJIA risk or pathophysiology. The strongest sJIA risk locus identified by this study was the MHC locus on chromosome 6 (see online supplementary figure S3). We have recently described this association in great detail in the context of a regional association study of the MHC locus in sJIA. ${ }^{14}$ Beyond the MHC locus, we identified a novel sJIA susceptibility locus on the short arm of chromosome 1 (1p36.32) whose association also exceeded the threshold for genome-wide significance under the additive model (figures 1 and 2). This locus includes a cluster of 14 sJIA-associated SNPs that span $20.6 \mathrm{~kb}$; the peak $\mathrm{SNP}$ is $\mathrm{rs} 72632736\left(\mathrm{p}=2.9 \times 10^{-9}\right.$; OR $2.4(1.8,3.3)$. The association peak is located $20 \mathrm{~kb}$ upstream of LOC284661, a long intergenic non-coding RNA, and $263.5 \mathrm{~kb}$ upstream of the nearest protein coding gene, AJAP1, encoding adherens junction-associated protein 1. Examination of ENCODE (Encyclopedia of Noncoding DNA Elements) data revealed that the sJIA-associated SNPs overlaid a cluster of transcription factor-binding sites (TFBS) identified by chromatin immunoprecipitation sequencing (ChIP-seq; figure 2) in a variety of cell types; however, none of the top sJIA-associated SNPs were located within the ChIP-seq TFBS.

In addition to the two loci described above, this study identified 23 novel candidate susceptibility loci (figure 1, table 2),

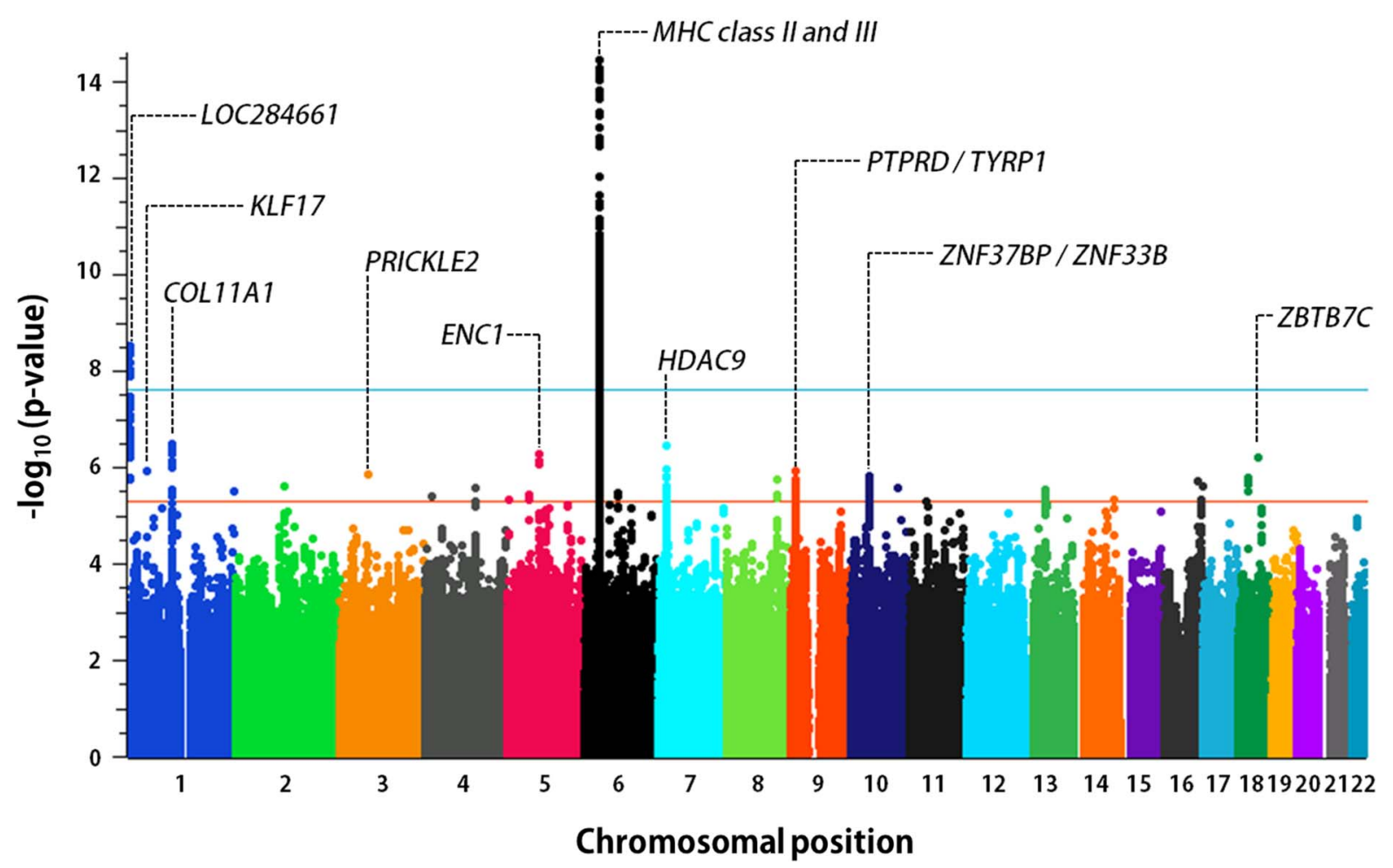

Figure 1 Genome-wide association results from meta-analysis of nine INCHARGE sJIA collections. The threshold of genome-wide significance $\left(p<2.5 \times 10^{-8}\right)$ is shown by the blue line, while the orange line marks the level of significance suggestive of association $\left(p<5 \times 10^{-6}\right)$. The top 10 sJIA-associated loci are labelled with the name of the nearest gene(s). INCHARGE, International Childhood Arthritis Genetics Consortium; MHC, major histocompatibility complex; sJIA, systemic juvenile idiopathic arthritis. 
Table 2 Susceptibility loci with at least suggestive evidence of association with sJIA

\begin{tabular}{|c|c|c|c|c|c|c|c|c|c|c|}
\hline Top SNP & $\mathrm{Chr}$ & Position & Ref/Alt & Best $p$ Value & Model & $\mathrm{OR}(\mathrm{Cl})$ & $\mathrm{i}^{2}$ & Strata & Samples & Closest gene(s) \\
\hline rs41291794 & 6 & 32425762 & $\mathrm{~A} / \mathrm{T}$ & $3.6 \times 10^{-15}$ & Additive & 2.1 (1.8 to 2.6$)$ & 0.64 & 9 & 7711 & HLA-DRA \\
\hline rs72632736 & 1 & 4449204 & $\mathrm{~A} / \mathrm{G}$ & $2.9 \times 10^{-9}$ & Additive & $2.4(1.8$ to 3.3$)$ & 0 & 7 & 7075 & LOC284661, AJAP1 \\
\hline rs1823549 & 1 & 103147831 & $\mathrm{~T} / \mathrm{C}$ & $3.2 \times 10^{-7}$ & Additive & $0.4(0.3$ to 0.6$)$ & 0 & 6 & 6816 & COL11A1 \\
\hline rs1178121 & 7 & 18762652 & $\mathrm{C} / \mathrm{A}$ & $3.4 \times 10^{-7}$ & Dominant & $1.6(1.3$ to 1.9$)$ & 0.24 & 8 & 7513 & HDAC9 \\
\hline rs12517545 & 5 & 73680314 & $\mathrm{G} / \mathrm{A}$ & $5.2 \times 10^{-7}$ & Dominant & $0.6(0.5$ to 0.8$)$ & 0 & 9 & 7711 & ENC1, LOC101929082 \\
\hline rs79575701 & 18 & 45579621 & $\mathrm{C} / \mathrm{A}$ & $6.2 \times 10^{-7}$ & Additive & $3.4(2.1$ to 5.5$)$ & 0 & 4 & 4822 & ZBTB7C \\
\hline rs114940806 & 1 & 44558672 & $\mathrm{~A} / \mathrm{G}$ & $1.2 \times 10^{-6}$ & Additive & $3.0(1.9$ to 4.7$)$ & 0.47 & 5 & 5137 & KLF17 \\
\hline rs1279094 & 9 & 11706771 & $\mathrm{~T} / \mathrm{C}$ & $1.2 \times 10^{-6}$ & Additive & $1.3(1.2$ to 1.5$)$ & 0 & 9 & 7712 & LOC101929446 \\
\hline rs864089 & 3 & 64244118 & $\mathrm{~T} / \mathrm{C}$ & $1.4 \times 10^{-6}$ & Dominant & $0.6(0.5$ to 0.8$)$ & 0 & 8 & 7516 & PRICKLE2 \\
\hline rs481331 & 10 & 43003048 & $\mathrm{~A} / \mathrm{T}$ & $1.4 \times 10^{-6}$ & Additive & 1.5 (1.3 to 1.7$)$ & 0 & 9 & 7712 & ZNF37BP, ZNF33B \\
\hline rs8097070 & 18 & 23086307 & $\mathrm{~A} / \mathrm{G}$ & $1.6 \times 10^{-6}$ & Additive & $0.3(0.2$ to 0.5$)$ & 0 & 4 & 4993 & ZNF521, SS18 \\
\hline rs1527934 & 8 & 117392156 & $\mathrm{C} / \mathrm{T}$ & $1.8 \times 10^{-6}$ & Additive & $1.7(1.4$ to 2.1$)$ & 0 & 6 & 6926 & EIF3H, LINC00536 \\
\hline rs78507369 & 16 & 78305293 & $A / G$ & $2.0 \times 10^{-6}$ & Additive & $3.0(1.9$ to 4.6$)$ & 0 & 4 & 4857 & WWOX, LSM3P5 \\
\hline rs12445022 & 16 & 87575332 & $\mathrm{G} / \mathrm{A}$ & $2.4 \times 10^{-6}$ & Dominant & 1.5 (1.3 to 1.7$)$ & 0.36 & 9 & 7715 & LOC101928737, JPH3 \\
\hline rs112165031 & 2 & 112902227 & $\mathrm{G} / \mathrm{A}$ & $2.5 \times 10^{-6}$ & Additive & 2.5 (1.7 to 3.7$)$ & 0.58 & 5 & 6917 & FBLN7 \\
\hline rs6853094 & 4 & 116576274 & $\mathrm{C} / \mathrm{A}$ & $2.6 \times 10^{-6}$ & Additive & $1.6(1.3$ to 1.9$)$ & 0.22 & 8 & 7564 & RPF2P2, PGAM4P2 \\
\hline rs73401585 & 10 & 109690236 & $\mathrm{~T} / \mathrm{C}$ & $2.6 \times 10^{-6}$ & Additive & $3.2(2.0$ to 5.2$)$ & 0 & 4 & 4824 & LOC101927573, SORCS1 \\
\hline rs9595973 & 13 & 49286438 & $\mathrm{G} / \mathrm{A}$ & $2.8 \times 10^{-6}$ & Dominant & $2.8(1.8$ to 4.3$)$ & 0.5 & 4 & 6845 & CYSLTR2 \\
\hline rs9633402 & 1 & 247946160 & $\mathrm{G} / \mathrm{A}$ & $3.0 \times 10^{-6}$ & Dominant & $0.4(0.2$ to 0.6$)$ & 0 & 9 & 7708 & TRIM58 \\
\hline rs62438583 & 6 & 75326244 & $T / G$ & $3.4 \times 10^{-6}$ & Dominant & $0.7(0.6$ to 0.8$)$ & 0 & 9 & 7712 & LOC101928516, COL12A1 \\
\hline rs62359376 & 5 & 52411328 & $\mathrm{G} / \mathrm{A}$ & $3.6 \times 10^{-6}$ & Dominant & $1.7(1.4$ to 2.2$)$ & 0.13 & 8 & 7516 & LOC257396, MOCS2 \\
\hline rs1501138 & 4 & 16397067 & $\mathrm{~T} / \mathrm{C}$ & $4.0 \times 10^{-6}$ & Dominant & $0.3(0.2$ to 0.5$)$ & 0.24 & 8 & 7517 & LDB2, TAPT1, ZEB2P1 \\
\hline rs7712113 & 5 & 4985443 & $\mathrm{G} / \mathrm{C}$ & $4.5 \times 10^{-6}$ & Dominant & 3.7 (2.1 to 6.5$)$ & 0.68 & 4 & 4661 & LINC01020, LOC101929176 \\
\hline rs1885747 & 14 & 93047455 & $A / G$ & $4.6 \times 10^{-6}$ & Additive & $1.4(1.2$ to 1.7$)$ & 0.38 & 8 & 7513 & RIN3, LGMN \\
\hline rs111580313 & 16 & 86621219 & $\mathrm{C} / \mathrm{T}$ & $4.8 \times 10^{-6}$ & Dominant & 1.7 (1.4 to 2.2$)$ & 0 & 7 & 7368 & MTHFSD, FOXL1, FOXC2 \\
\hline
\end{tabular}

Best $p$ value, meta-analytic $p$ value corrected for gender and ancestry under the model specified in the Model column. Model, the genetic model (either additive or dominant) that showed the strongest association between the SNP and sJIA. $I^{2}, I^{2}$ test for heterogeneity. Strata, number of strata included in meta-analysis. Samples, number of samples included in meta-analysis.

Alt, alternate allele; Chr, chromosome; Ref, reference allele; sJIA, systemic juvenile idiopathic arthritis; SNP, single nucleotide polymorphism;

two of which are shown in detail in online supplementary figure S4. Importantly, the top 25 sJIA susceptibility loci had scant intersection with the known susceptibility loci of other JIA subtypes. Based on this observation, we sought to compare the genetic architecture of sJIA with those of polygoJIA and RF + polyJIA.

We first examined the 23 polygoJIA-associated loci reported by Hinks $e t a l^{12}$ in the sJIA study population and none showed even a modest association with sJIA (see online supplementary tables S5 and S6). To more formally compare sJIA with polygoJIA, we calculated a polygo-wGRS in the sJIA casecontrol collections based on the same 23 SNPs. The nonparametric Wilcoxon rank-sum test found no difference in the distribution of polygoJIA risk allele counts or polygo-wGRSs between sJIA cases and controls (figure 3, see online supplementary table S7 and figures S5 and S6). Consistent with this, logistic regression analysis found no correlation between the polygo-wGRS and sJIA in any individual stratum or in the full study population (see online supplementary table S7). Analysis of ROC curves in individual strata and the full population found that the AUCs for polygo-wGRS were all close to 0.5, indicating that the polygo-wGRS was no better than random chance at distinguishing sJIA cases from control subjects (figure 3, see online supplementary figure S7). Finally, to expand the scope of our comparison beyond peak SNPs from risk loci, we performed a Q-Q plot-based enrichment analysis to look for shared genetic risk factors between sJIA and
polygoJIA (figure 3). By comparing Q-Q plots of polygoJIA-associated $\mathrm{SNPs}^{12}$ at several different significance levels in our sJIA collection, we sought to evaluate pleiotropy in a more global/genomic manner. In the presence of pleiotropy, the slopes of the $\mathrm{Q}-\mathrm{Q}$ plots of disease A associations are expected to increase as the plotted SNP sets become more strongly associated with disease $\mathrm{B}$, as previously shown. ${ }^{20}$ In the case of polygoJIA-associated SNPs in sJIA, the slope of the Q-Q plots of sJIA associations did not increase when SNPs of increasingly strong association with polygoJIA were plotted, indicating that there was no enrichment of sJIA-associated variants among polygoJIA-associated variants, and therefore that there was no evidence of pleiotropy (figure 3 ).

In addition, we used an RF+ poly-wGRS ${ }^{19}$ to look for shared genetic architecture between sJIA and RF+polyJIA. As was the case with polygoJIA, non-parametric testing revealed no significant difference in the distribution of RF+polyJIA risk alleles (see online supplementary figure S8) or RF+poly-wGRS (see online supplementary figure S9) between sJIA cases and controls in any individual population. Of note, non-parametric testing and logistic regression analysis identified a significant difference in RF+ poly-wGRS between sJIA and controls in the full collection (see online supplementary table S8 and figure S10); however, the wGRSs were actually lower in the sJIA cases than in the controls (see online supplementary figure S11). Consistent with these observations, ROC analyses found that the $\mathrm{RF}+$ poly-wGRS was not predictive of sJIA (see online 


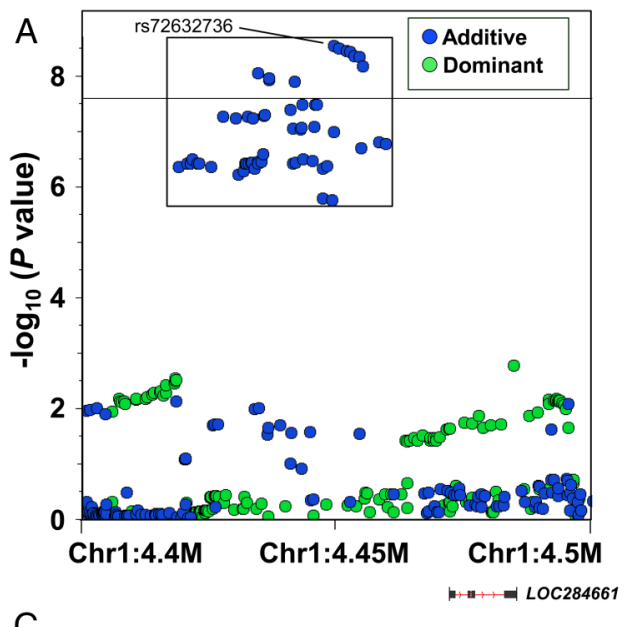

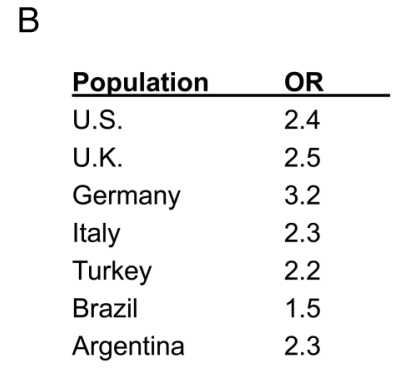

Meta Analysis $2.4(1.8,3.3)$

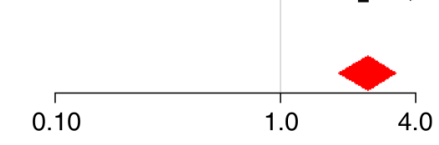

C
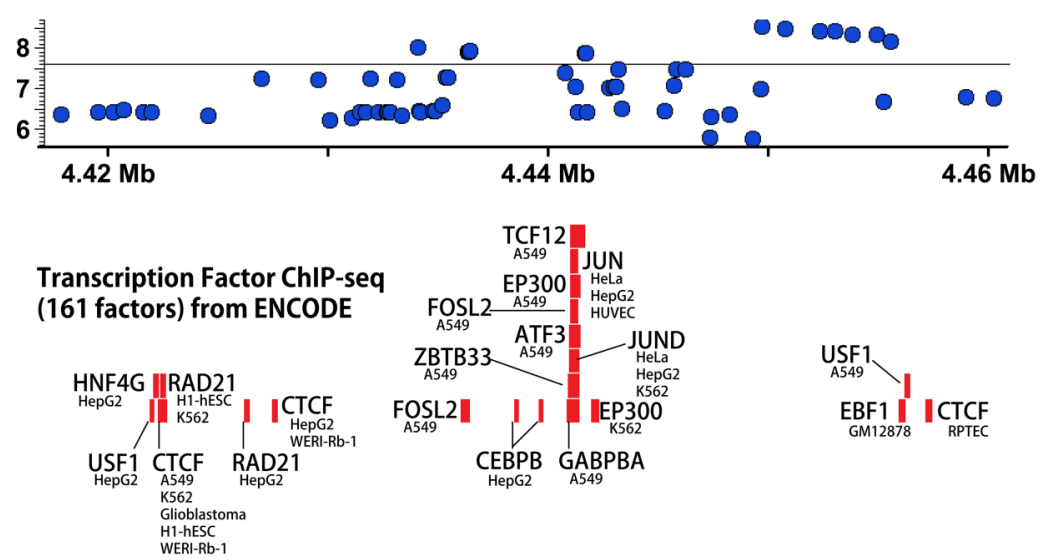

Figure 2 Systemic juvenile idiopathic arthritis (sJA) susceptibility locus at chr1p36.32. A regional association plot demonstrates the association between SJIA) and single nucleotide polymorphisms (SNPS) in this region (A). The effect of the peak SNP (rs72632736) in each study population is demonstrated in the forest plot (B). The threshold of genome-wide significance $\left(p<2.5 \times 10^{-8}\right)$ is marked by the black horizontal line in (A) and (C). Panel C shows the superimposition of sJA-associated SNPs (inset box, A) with transcription factor-binding sites determined by chromatin immunoprecipitation (ChIP) sequencing from the Encyclopedia of Noncoding DNA Elements (ENCODE) project.

supplementary figures S10 and S12). Collectively, these investigations failed to identify any evidence of shared genetic architecture between sJIA and polygoJIA or RF + polyJIA.

\section{DISCUSSION}

In this study, two novel susceptibility loci met genome-wide significance criteria for association with sJIA and 23 other loci demonstrated highly suggestive evidence of association. Furthermore, formal comparisons of association data from sJIA with those from polygoJIA and RF+ polyJIA have demonstrated that sJIA bears a unique genetic architecture, indicating that its underlying pathophysiological mechanisms are significantly divergent from other forms of JIA. This has important implications and should direct research for future targets of therapeutic intervention for children affected with sJIA.

This is the first large-scale genomic study of sJIA, which includes case-control collections from nine different countries. In a sample of 982 affected children, we identified genome-wide significant evidence of association with SNPs in the class II MHC locus and SNPs on chromosome 1 nearest to an uncharacterised long non-coding RNA gene. This work also identified many additional candidate sJIA susceptibility loci, nearly all of them novel, and aside from the HLA locus, none of these novel loci are associated with any other rheumatic diseases (see online supplementary table S9). The identification of these loci is an important step towards the elucidation of the specific pathways and pathogenic mechanisms in sJIA, which in turn will allow the development of therapies to more specifically target sJIA pathophysiology in affected children. Several of the susceptibility loci that warrant further investigation include strong candidates for therapeutic modulation, and many novel loci or genes that have been poorly studied, to date. Functional investigations are needed to identify and understand the specific mechanisms that underlie the genetic associations.

This study also provided the first opportunity to demonstrate that sJIA did not share heritable risk factors with the more common oligoarticular and polyarticular forms of JIA. There was no intersection of the top susceptibility loci of sJIA with those of polygoJIA or RF+polyJIA. Even within the class II MHC region, which harbours disease-associated genetic variation in each of these categories of JIA, the subtype-specific risk factors (SNPs, HLA alleles and HLA haplotypes) are not shared between subtypes. Using a combination of genetic risk scores and enrichment analysis, this study reveals an absence of shared genetic architecture between sJIA and either polygoJIA or RF + polyJIA, despite often sharing a chronic arthritis feature with polygo or RF+polyJIA. It could be that as a clinical feature, arthritis is a non-specific finding that is present in many different conditions, including infections, malignancies, autoimmune disorders and autoinflammatory conditions. These distinct genetic data provide hard evidence that these conditions differ in pathophysiology, strongly supporting the clinical distinction 
A
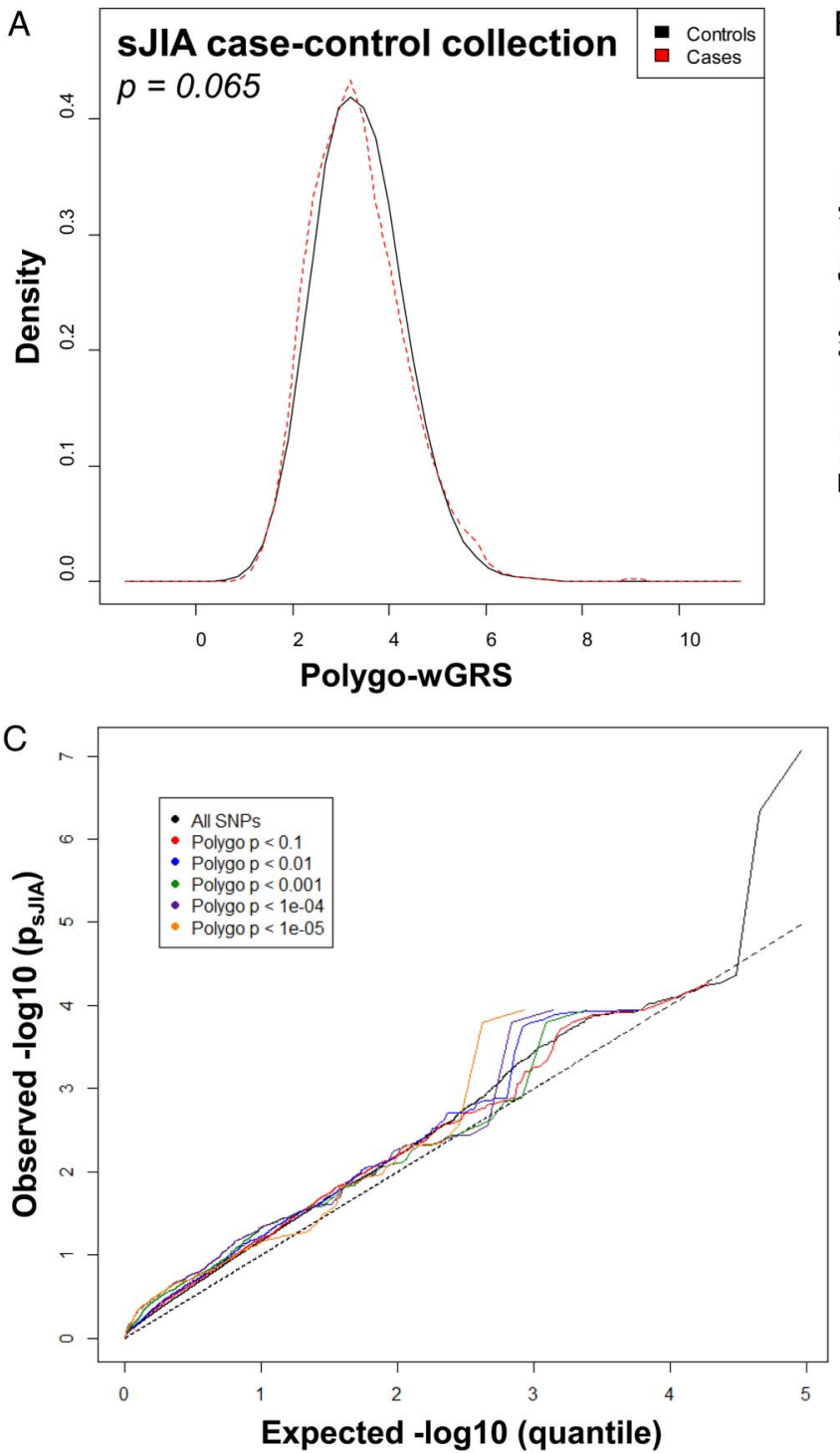

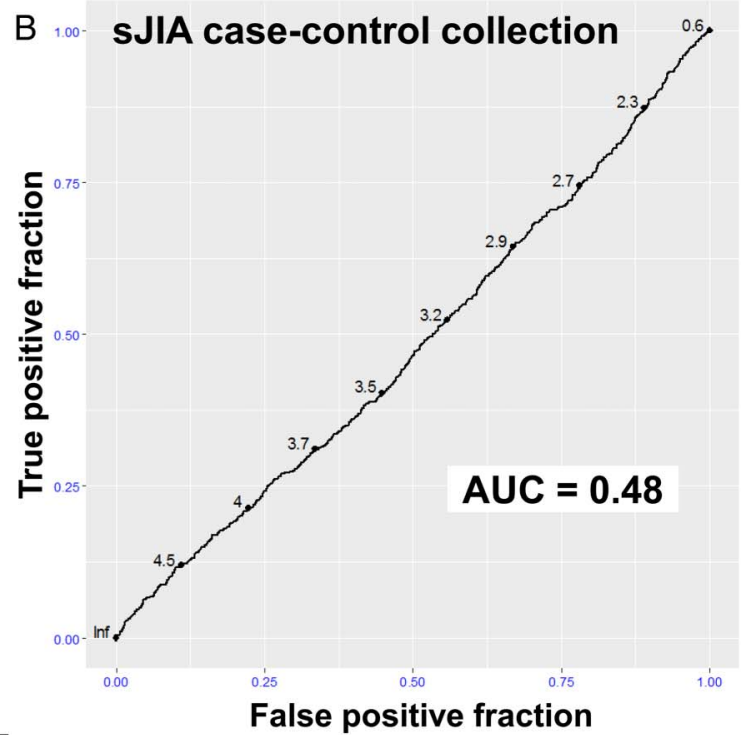

False positive fraction between sJIA and the other JIA subtypes. Considering the ongoing discussions about restructuring the JIA nomenclature, these studies will help inform and guide the debate surrounding sJIA ${ }^{7}$ and how it should be classified.

The genetic dissimilarity of sJIA and other JIA subtypes has important therapeutic implications for children with sJIA. Currently, the treatment of sJIA presents physicians with a clinical conundrum, with no single, universally effective therapeutic approach. Prior to the era of biological response-modifying agents, sJIA was treated with disease-modifying antirheumatic drugs, including methotrexate, with a rationale for use extrapolated from other forms of JIA; there were no clinical trials and only limited outcome studies describing their effectiveness in sJIA. ${ }^{10}$ In the absence of clear therapeutic alternatives, and despite the limited evidence of efficacy, methotrexate remains an accepted therapeutic option in the consensus treatment protocol. ${ }^{21}$ Similarly, therapies targeting the cytokine tumour necrosis factor- $\alpha$ are highly effective in the treatment of other forms of
JIA, ${ }^{22}$ but show only modest effect in children with sJIA. ${ }^{10}$ Today, even with the most effective treatments for sJIA directed against the inflammatory cytokines interleukin (IL)-1 and IL- $6,{ }^{10}$ a sizable proportion of children continue to have active disease, with chronic arthritis persisting in nearly $40 \%$ of children in a recent study. ${ }^{9}$ Currently, the only widely effective treatment for sJIA remains large doses of glucorticoids. ${ }^{10}$ There is clearly an imperative to look for root causes of sJIA to identify better targets for therapy and prevent the development of persistent, disabling arthritis.

Although it is necessary to better understand the function of the risk alleles identified by this study, the results may identify genetic profiles that can be used to determine appropriate therapeutic interventions. To this point, two susceptibility loci are of particular therapeutic interest in sJIA: the class II HLA locus and HDAC9, encoding histone deacetylase 9. Given that class II HLA molecules present peptide antigens to T-cell receptors on CD4+ T cells, resulting in their activation, one may predict that 
therapeutic modulation of T-cell activation would be an effective strategy in the treatment of sJIA. In fact, abatacept, which reduces T-cell activation through costimulatory inhibition, has shown promising results in children with the chronic, persistent arthritis of SJIA $^{23} 24-$ a subset of patients with sJIA who are particularly refractory to therapeutic intervention. ${ }^{5}$ Based on these observations, it may be reasonable to use abatacept in children with sJIA. HDAC9 confers important epigenetic effects through deacetylation of histone proteins, while also regulating critical innate immune processes, including Toll-like receptor signalling and the development of regulatory $\mathrm{T}$ cells, via deacetylation of non-histone targets. ${ }^{25-28}$ Despite the fact that HDAC9 was only suggestively associated with sJIA, a pilot study of the nonspecific HDAC inhibitor, gavinostat, produced promising preliminary results in children with $\mathrm{sJIA},{ }^{29}$ raising the possibility that HDAC inhibition represents another plausible targeted therapeutic strategy in sJIA.

At a time when an emphasis is being placed on the personalisation of medicine, it is important that we move away from broad classifications based on non-specific clinical observations and move towards the use of molecular and genetic data in establishing diagnoses, as well as pathophysiology. In turn, clinical practice will advance as these data are translated into targeted therapeutic approaches. Perhaps it is time to separate this condition from JIA all together to make clear that it is fundamentally different from any other form of JIA and needs to be considered and treated differently. Given that the currently available treatments for this condition are still imperfect, it remains imperative to continue to employ contemporary investigative approaches in sJIA, to elucidate its pathophysiology and to identify the next generation of therapeutic strategies.

\footnotetext{
Author affiliations

${ }^{1}$ Translational Genetics and Genomics Unit, National Institute of Arthritis and Musculoskeletal and Skin Diseases, National Institutes of Health, US Department of Health and Human Services, Bethesda, Maryland, USA

${ }^{2}$ Inflammatory Disease Section, National Human Genome Research Institute, National Institutes of Health, US Department of Health and Human Services, Bethesda, Maryland, USA

${ }^{3}$ Arthritis Research UK Centre for Genetics and Genomics, Centre for Musculoskeletal Research, Manchester Academic Health Science Centre, Manchester, UK

${ }^{4}$ Human Genetics, The Wellcome Trust Sanger Institute, Hinxton, UK ${ }^{5}$ Department of Pediatrics, University of Cincinnati College of Medicine, Cincinnati, Ohio, USA

${ }^{6}$ Cincinnati Children's Hospital Medical Center, Cincinnati, Ohio, USA

'Department of Pediatric Rheumatology and Immunology, University Hospital Münster, Münster, Germany

${ }^{8}$ Department of Pediatrics, University of Genova, Genoa, Italy

${ }^{9}$ Pediatrics II Unit, Giannina Gaslini Institute, Genoa, Italy

${ }^{10}$ Department of Pediatric Rheumatology, Hacettepe University, Ankara, Turkey

${ }^{11}$ Departments of Pediatrics and Human Genetics, Emory University School of Medicine, Atlanta, Georgia, USA

${ }^{12}$ Children's Healthcare of Atlanta, Atlanta, Georgia, USA

${ }^{13}$ Department of Pediatrics, Cleveland Clinic, Cleveland, Ohio, USA

${ }^{14}$ Department of Pediatrics, University of Utah, Salt Lake City, Utah, USA

${ }^{15}$ Department of Pediatrics, Albert Einstein College of Medicine and Children's Hospital at Montefiore, Bronx, New York, USA

${ }^{16}$ Department of Pediatrics, Stanford University, Stanford, California, USA

${ }^{17}$ Service of Immunology and Rheumatology, Hospital de Pediatria Garrahan, Buenos Aires, Argentina

${ }^{18}$ Department of Pediatrics, Universidade Federal de São Paulo, São Paulo, Brazil

${ }^{19}$ Universidade Federal de Rio de Janeiro, Rio de Janeiro, Brazil

${ }^{20}$ Department of Pediatrics, University of Toronto, Toronto, Canada

${ }^{21}$ Department of Immunology, University of Toronto, Toronto, Canada

${ }^{22}$ Institute of Medical Science, University of Toronto, Toronto, Canada

${ }^{23}$ Department of Pediatrics, University of Saskatchewan, Saskatoon, Canada

${ }^{24}$ Institute of Child Health, University College London, London, UK

${ }^{25}$ Center of Paediatric and Adolescent Rheumatology, University College London,

London, UK
}

${ }^{26}$ Pediatric Rheumatology Unit, Hospital Sant Joan de Déu, Universitat de Barcelona, Barcelona, Spain

${ }^{27}$ German Center for Pediatric and Adolescent Rheumatology, GarmischPartenkirchen, Germany

${ }^{28}$ University Hospital Cal Gustav Carus, Dresden, Germany

${ }^{29}$ Department of Rheumatology and Clinical Immunology, Charité -University Medicine, Berlin, Germany

${ }^{30}$ Epidemiology Unit, German Rheumatism Research Centre, Berlin, Germany

${ }^{31}$ Department of Pediatrics, RWTH Aachen University, Aachen, Germany

${ }^{32}$ National Institute for Health Research Manchester Musculoskeletal Biomedical

Research Unit, Central Manchester University Hospitals NHS Foundation Trust, Manchester Academic Health Science Centre, University of Manchester, Manchester, UK

${ }^{33}$ Department of Medicine, University of Pittsburgh, Pittsburgh, Pennsylvania, USA

${ }^{34}$ Department of Human Genetics, University of Pittsburgh, Pittsburgh, Pennsylvania, USA

${ }^{35}$ Department of Gastroenterology and Hepatology, Cleveland Clinic, Cleveland, Ohio, USA

${ }^{36}$ Department of Pathobiology, Cleveland Clinic, Cleveland, Ohio, USA

${ }^{37}$ Department of Genetics and Genomic Sciences, Icahn School of Medicine at

Mount Sinai, New York, New York, USA

${ }^{38}$ The Centre for Applied Genomics, The Hospital for Sick Children, Toronto, Ontario, Canada

${ }^{39}$ Center for Genomics and Oncological Research, Pfizer-University of GranadaAndalusian Government, Granada, Spain

${ }^{40}$ Unit of Chronic Inflammatory Diseases, Institute for Environmental Medicine, Karolinska Institutet, Solna, Sweden

${ }^{41}$ Interdisciplinary Cluster for Applied Genoproteomics-Université de Liège, Liège, Belgium

${ }^{42}$ Centre for Genomic Regulation (CRG), The Barcelona Institute of Science and Technology, and Universitat Pompeu Fabra (UPF), Barcelona, Spain

${ }^{43}$ Sidra Medical and Research Centre, Doha, Qatar

${ }^{44}$ Istanbul Faculty of Medicine, Istanbul University, Istanbul, Turkey

${ }^{45}$ Department of Biostatistical Sciences, Wake Forest University Health Sciences,

Winston-Salem, North Carolina, USA

Twitter Follow NIAMS/NIH/DHHS @NIH_NIAMS; Ricardo Russo @el_reumatologo; University of Manchester, Centre for Musculoskeletal Disease @UoMMskResearch

Collaborators Full membership of collaborating consortia are listed in the supplementary text: British Society of Pediatric and Adolescent Rheumatology Study Group, Inception Cohort of Newly Diagnosed Patients with Juvenile Idiopathic Arthritis Study Group, Childhood Arthritis Prospective Study Group, Randomized Placebo Phase Study of Rilonacept in sJIA Investigators,

Sparks-Childhood Arthritis Response to Medication Study Group and Biologically Based Outcome Predictors in JIA Group.

Contributors All authors participated in study design; AAG, DF, AM, MG, SÖ, SP, ASZ, JFB, NTI, EDM, RR, CL, MOEH, SO, RSMY, AMR, LRW, JA, J-PH, AR-W, KM, $K T, E D, B S P A R, ~ I C O N-J I A$, CAPS, RAPPORT, CHARMS, BBOP, RHD, JPA, MIK, KMK, LCK, DP, SWS, MEA-R, ED, XE and AG provided samples for the study; MJO, VLA, EFR, AH, IT, EZ, PW and WT performed the research and analysis and interpreted the data; all authors drafted and/or substantively edited the manuscript and have thoroughly reviewed and approved of the content.

Funding This work was supported by the Intramural Research Programs of the National Institute of Arthritis and Musculoskeletal and Skin Diseases

(Z01-AR041198 to MJO) and the National Human Genome Research Institute (Z01-HG200370 to DLK) of the National Institutes of Health (NIH). Additional funding was provided by NIH grants R01-AR059049 (AAG), R01AR061297 (EDM), R01-AR060893 (SP), P30-AR47363 and P01-AR48929 (ST), AG030653, AG041718 and AG005133 (MIK) and U01-DK062420 and R01-DK076025 (RHD); Arthritis Research UK Grant 20385 (WT); the German Federal Ministry of Education and Research (BMBF project 01ER0813) for the 'ICON-JIA' inception cohort (KM and DF); the Val A. Browning Charitable Foundation (JFB); the Marcus Foundation (SP); the Proyecto de Excelencia (CTS-2548) of the Andalousian Government (MA-R) and the Swedish Association Against Rheumatism (MA-R). IT and EZ were supported by the Wellcome Trust (098051). WT and JC are funded by the National Institute for Health Research Biomedical Research Unit Funding Scheme. The views expressed in this publication are those of the author(s) and not necessarily those of the NHS, the National Institute for Health Research or the Department of Health. The CAPS study was funded by Arthritis Research UK Grant 20542. WT, AH, and JC are supported by the Manchester Academic Health Sciences Centre (MAHSC). SPARKS-CHARMS was funded by grants from SPARKS UK (08ICH09 and 12ICH08), the Medical Research Council (MR/M004600/1) and the UK National Institute for Health Research GOSH Biomedical Research Centre. The BBOP study was supported by the Canadian Institutes of Health Research and the Arthritis Society (CIHR funding reference number 82517) and the Canadian Arthritis Network (funding reference SRI-IJD-01). This research was supported in part by the Cincinnati Children's 
Research Foundation and its Cincinnati Genomic Control Cohort. The authors acknowledge the use of DNA from the UK Blood Services collection of Common Controls (UKBS-CC collection), which is funded by Wellcome Trust grant 076113/C/ $04 / Z$ and by the USA NIH research programme grant to the National Health Service Blood and Transplant (RP-PG-0310-1002). The authors acknowledge the use of DNA from the British 1958 Birth Cohort collection, which is funded by the UK Medical Research Council grant G0000934 and the Wellcome Trust grant 068545/Z/ 02. This study used the computational resources of the Biowulf system at the $\mathrm{NIH}$ Bethesda, MD (http://biowulf.nih.gov).

Competing interests AAG: consulting fees and honoraria from Novimmune, Novartis, Roche. AM: consulting fees and honoraria from Abbvie, Boehringer, Celgene, CrescendoBio, Janssen, Meddimune, Novaris, NovoNordisk, Pfizer, Sanofi Aventis, Vertex and Servier, contributions have been received by G. Gaslini Hospital and the PRINTO network by BMS, GlaxoSmithKline, Hoffman-La Roche, Novartis, Pfizer, Sanofi Aventis, Schwarz Biosciences, Abbot, Francesco Angelini S.P.A., Sobi, and Merck Serono. MG: consulting and speaker fees from SOBI and Novartis, unrestricted grants to Eurofever from SOBI and Novartis. SP: consulting fees from Novartis. EDM: consulting fees from Novartis. LRW: speaker fees from Pfizer.

Ethics approval University of Manchester.

Provenance and peer review Not commissioned; externally peer reviewed.

Data sharing statement The quality control processed directly genotyped SNP genotype data from $\mathrm{SJIA}$ cases genotyped for this study will be deposited into the National Institutes of Health's Database of Genotypes and Phenotypes, where allowable by the Ethics and consent documents. The future use of these data will be dictated by the terms of Ethics and consent documents and the institutional certifications provided by the collaborating centres.

Open Access This is an Open Access article distributed in accordance with the Creative Commons Attribution Non Commercial (CC BY-NC 4.0) license, which permits others to distribute, remix, adapt, build upon this work non-commercially, and license their derivative works on different terms, provided the original work is properly cited and the use is non-commercial. See: http://creativecommons.org/ licenses/by-nc/4.0/

\section{REFERENCES}

1 Prakken B, Albani S, Martini A. Juvenile idiopathic arthritis. Lancet 2011;377:2138-49

2 Petty RE, Southwood TR, Manners P, et al. International League of Associations for Rheumatology classification of juvenile idiopathic arthritis: second revision, Edmonton, 2001. J Rheumatol 2004;31:390-2.

3 Bannatyne GA, Wohlmann AS. Rheumatoid arthritis: It's clinical history, etiology, and pathology. Lancet 1896;147:1120-5.

4 Still GF. On a form of chronic joint disease in children. Med Chir Trans 1897;80:47-60.

5 Mellins ED, Macaubas C, Grom AA. Pathogenesis of systemic juvenile idiopathic arthritis: some answers, more questions. Nat Rev Rheumatol 2011;7:416-26.

6 Davies R, Southwood T, Kearsley-Fleet L, et al. Standardized mortality rates are increased in patients with severe juvenile idiopathic arthritis. Rheumatology 2015;54:153.

7 Martini A. It is time to rethink juvenile idiopathic arthritis classification and nomenclature. Ann Rheum Dis 2012;71:1437-9.

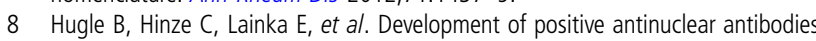
and rheumatoid factor in systemic juvenile idiopathic arthritis points toward an autoimmune phenotype later in the disease course. Pediatr Rheumatol Online J 2014;12:28.

9 Janow G, Schanberg LE, Setoguchi $S$, et al. The systemic juvenile idiopathic arthritis cohort of the childhood arthritis and rheumatology research alliance registry: 2010-2013. J Rheumatol 2016;43:1755-62.

10 Beukelman T. Treatment advances in systemic juvenile idiopathic arthritis. F1000Prime Rep 2014;6:21.

11 Solovieff $\mathrm{N}$, Cotsapas C, Lee PH, et al. Pleiotropy in complex traits: challenges and strategies. Nat Rev Genet 2013;14:483-95.

12 Hinks A, Cobb J, Marion MC, et al. Dense genotyping of immune-related disease regions identifies 14 new susceptibility loci for juvenile idiopathic arthritis. Nat Genet 2013:45:664-9.

13 Thompson SD, Marion MC, Sudman M, et al. Genome-wide association analysis of juvenile idiopathic arthritis identifies a new susceptibility locus at chromosomal region 3q13. Arthritis Rheum 2012;64:2781-91.

14 Ombrello MJ, Remmers EF, Tachmazidou I, et al. HLA-DRB1*11 and variants of the MHC class II locus are strong risk factors for systemic juvenile idiopathic arthritis. Proc Natl Acad Sci USA 2015;112:15970-5.

15 Marchini J, Howie B, Myers $S$, et al. A new multipoint method for genome-wide association studies by imputation of genotypes. Nat Genet 2007;39:906-13.

16 Howie B, Marchini J, Stephens M. Genotype imputation with thousands of genomes. G3 (Bethesda) 2011;1:457-70.

17 Magi R, Morris AP. GWAMA: software for genome-wide association meta-analysis. BMC Bioinformatics 2010;11:288.

18 Karlson EW, Chibnik LB, Kraft P, et al. Cumulative association of 22 genetic variants with seropositive rheumatoid arthritis risk. Ann Rheum Dis 2010;69:1077-85.

19 Prahalad S, Conneely KN, Jiang Y, et al. Susceptibility to childhood-onset rheumatoid arthritis: investigation of a weighted genetic risk score that integrates cumulative effects of variants at five genetic loci. Arthritis Rheum 2013;65:1663-7.

20 Liley J, Wallace C. A pleiotropy-informed Bayesian false discovery rate adapted to a shared control design finds new disease associations from GWAS summary statistics. PLoS Genet 2015:11:e1004926.

21 DeWitt EM, Kimura Y, Beukelman T, et al. Consensus treatment plans for new-onset systemic juvenile idiopathic arthritis. Arthritis Care Res (Hoboken) 2012;64:1001-10.

22 Ungar WJ, Costa V, Burnett HF, et al. The use of biologic response modifiers in polyarticular-course juvenile idiopathic arthritis: a systematic review. Semin Arthritis Rheum 2013;42:597-618.

23 Ruperto N, Lovell DJ, Quartier $\mathrm{P}$, et al. Abatacept in children with juvenile idiopathic arthritis: a randomised, double-blind, placebo-controlled withdrawal trial. Lancet 2008;372:383-91.

24 Record JL, Beukelman T, Cron RQ. Combination therapy of abatacept and anakinra in children with refractory systemic juvenile idiopathic arthritis: a retrospective case series. J Rheumatol 2011:38:180-1.

25 Birnbaum RY, Clowney EJ, Agamy 0, et al. Coding exons function as tissue-specific enhancers of nearby genes. Genome Res 2012;22:1059-68.

26 Li X, Zhang Q, Ding Y, et al. Methyltransferase Dnmt3a upregulates HDAC9 to deacetylate the kinase TBK1 for activation of antiviral innate immunity. Nat Immunol 2016;17:806-15.

27 Li B, Samanta A, Song X, et al. FOXP3 interactions with histone acetyltransferase and class II histone deacetylases are required for repression. Proc Natl Acad Sci USA 2007; 104:4571-6.

28 Clark RI, Tan SW, Pean CB, et al. MEF2 is an in vivo immune-metabolic switch. Cell 2013;155:435-47.

29 Vojinovic J, Damjanov N, D'Urzo C, et al. Safety and efficacy of an oral histone deacetylase inhibitor in systemic-onset juvenile idiopathic arthritis. Arthritis Rheum 2011;63:1452-8. 